\title{
FAULT DETECTION IN NONLINEAR SYSTEMS
}

\author{
Kondo Adjallah, Frédéric Kratz, Didier Maquin
}

\author{
Centre de Recherche en Automatique de Nancy \\ BP 40 - Rue du doyen Marcel Roubault \\ 54501 Vandoeuvre les Nancy Cedex - FRANCE \\ Phone : (33) $83503080 \quad$ Fax : (33) 83503096
}

\begin{abstract}
This communication deals with the problem of fault detection and localization for a wide class of nonlinear systems subjected to bounded nonlinearities. A dedicated nonlinear observer scheme (DNOS) for fault detection and identification of observable systems is proposed.
\end{abstract}

\section{INTRODUCTION}

State observation of nonlinear dynamical systems is becoming a growing topic of investigation in the specialized literature. The reconstruction of the time behaviour of state variables remains a major problem both in control theory and process diagnosis. Researchers attention is being particularly focused on the design of adaptive observers for on-line process states estimation. There is increasing awareness that ensuring robustness in performance requires simpler and stable adaptive observer schemes. Linear systems have received considerable attention [1], [2] leading to several stable adaptive observer systems [3], [4], [5], [6]. Linear observer systems involving unknown inputs have also been developed and analyzed [7]. Nevertheless, the design of asymptotically stable observers remains a hard task in the nonlinear case, even when the nonlinearities are fully known.

Several observer design approaches have been proposed in recent years for nonlinear systems. Walcott [8], [9] for instance, proposed a new type of observers for systems subjected to bounded nonlinearities or uncertainties. This type of observer does not necessitate exact knowledge of the system nonlinearities. Bastin [10] also describes an adaptive observer/identifier, for SISO observable nonlinear systems, capable of state estimation and parameter adaptation simultaneously. Marino [11] developed the same idea and proposed a simpler observer restricted however to a class of systems with constant unknown parameters. As a main result, the construction of an observer may be performed by finding suitable state space and output changes of coordinates to transform the nonlinear system into an observable form from which can be derived an observer with linear dynamics. Xia [12] and Krener [13] have given Lie algebraic and rank conditions for nonlinear process observability and observer existence purpose.

Simultaneously, the techniques of fault detection and isolation (FDI) are increasingly discussed in both research and applications. It is based on the use of analytical redundancy. The general procedure first generates the socalled residuals (i.e., faults accentuated functions) before proceeding to fault detection and isolation (i.e., determination of its location, duration, type, magnitude, source). The use of observers is among the well established concepts for linear systems [14]. For the general case of nonlinear systems, little has so far been achieved in the development of associated FDI observers [15], [16]. Solutions so far proposed are difficult to apply in real situations.

This note is organized into four sections. The first presents the observer design for nonlinear systems. The second considers dedicated observers while the third deals with the analysis of generated residuals. The last section is devoted to computational aspects and uses the numerical results to highlight the strategy employed to isolate faults.

\section{OBSERVER DESIGN}

We shall consider nonlinear systems of the form:

$$
\begin{array}{ll}
\dot{x}(t)=f(x(t), u(t)) & x \in R^{n}, u \in R^{m} \\
y(t)=C x(t) & y \in R^{p}
\end{array}
$$

where $R^{n}$, and $R^{p}$ are the state and the output spaces of the system, $C$ is a constant matrix of appropriate dimensions. It is assumed that for any input $u(t)$ and initial state $x_{0}$ the corresponding state trajectory $x(t)$ is defined for all $t$ and that $f$ is continuously differentiable. Proceeding by analogy to the classical observer in the linear case, we seek an observer of the following form:

$$
\begin{aligned}
& \dot{z}(t)=f(z(t), u(t))+R(u(t))(y(t)-C z(t)) \quad z \in R^{n} \\
& y_{e}(t)=C z(t)
\end{aligned}
$$

The idea is to select a continuous mapping $R(u(t))$ so that $z(t)$ becomes a state estimator of the process under consideration.

The state and output errors are respectively defined by:

$e(t)=x(t)-z(t)$
$r(t)=y(t)-y_{e}(t)$
$r(t)=C e(t)$

The state error is solution of the equation: 


$$
\dot{e}(t)=f(x(t), u(t))-f(z(t), u(t))-R(u(t)) C e(t)
$$

The matrix function $R(u(t))$ is chosen so that the state error $e(t)$ asymptotically decreases and approaches zero as $t$ tends to infinity. The error $e(t)$ is then considered to be in the neighbourhood of zero. By using (3a), a first order Taylor expansion of the function $f(x(t), u(t))$ in the neighbourhood of the estimated state trajectory $z(t)$ can then be obtained as follows:

$$
\begin{aligned}
& f(x, u)=f(z+e, u) \\
& f(x, u)=f(z, u)+\frac{\partial f(z, u)}{\partial z} e
\end{aligned}
$$

which, when substituted in (4), gives:

$$
\dot{e}=\left(\frac{\partial f(z, u)}{\partial z}-R(u) C\right) e
$$

The aim is to define the mapping $R(u)$ so that when $t$ approaches infinity the error tends to zero. For that, let us consider the Liapunov quadratic function:

$$
V(e)=\frac{1}{2} e^{T} P e
$$

where $P$ is a positive definite matrix. We require time derivative of $V(e)$ to be negative:

$$
\begin{aligned}
& \dot{V}(e)=e^{T} P \dot{e} \\
& \dot{V}(e)=e^{T} P\left(\frac{\partial f(z, u)}{\partial z}-R(u) C\right) e<0
\end{aligned}
$$

This condition insures that $e$ decreases exponentially to zero [17], [18]. Tsinias [19] proposed an algorithm for determining the gain $R(u)$ based on the assumption that $\operatorname{Ker}(C) \neq\{0\}$. The algorithm comprises two steps.

$\underline{\text { Step } 1}$ taking $e \in \operatorname{Ker}(C)$, equation (8) can be reduced to:

$$
\dot{V}(e)=e^{T} P \frac{\partial f(z, u)}{\partial z} e
$$

The problem is to find $P$ which ensures the condition:

$$
\dot{V}(e)=e^{T} P \frac{\partial f(z, u)}{\partial z} e<0
$$

Solving inequation (10) yields the value for $P$. For that purpose, the assumption $\operatorname{Ker}(C) \neq\{0\}$ yields:

$$
e=K \bar{e}
$$

where $K$ is right orthogonal to $C$. Substituting (11) into (10) gives:

$$
\dot{V}(e)=\bar{e}^{T} K^{T} P \frac{\partial f(z, u)}{\partial z} K \bar{e}
$$

making $V(e)$ negative gives the matrix $P$.

If $\operatorname{Ker}(C)$ is reduced to $\{0\}$, step 1 is bypassed and $P$ is taking equal to the identity matrix.

Step 2 With this $P$ matrix previously determined, we now allow $e \in R^{n}$ and try to determine $R(u)$ which verifies equation (8). For that, we observe that:

$$
\dot{V}(e) \leq\left|e^{T} P \frac{\partial f(z, u)}{\partial z} e\right|-e^{T} P R(u) C e<0
$$

Using the structure proposed by Tsinias for $R(u)$, that is:

$$
R(u)=c h(u) P^{-1} C^{T}
$$

where $h(u)$ is a real positive valued function and $c$ a real positive constant, we obtain:

$$
\dot{V}(e) \leq\left|e^{T} P \frac{\partial f(z, u)}{\partial z} e\right|-c h(u)\|C e\|^{2}<0
$$

The basic problem comes to determining an $\bar{h}(u)$ which satisfies the following inequality:

$$
\left|e^{T} P \frac{\partial f(z, u)}{\partial z} e\right|-\bar{h}(u)\|e\|^{2}<0
$$

If $\bar{h}(u)$ exists, we then look for $c$ which verifies (15) by letting:

$$
h(u)=\bar{h}(u)
$$

which leads to:

$$
\dot{V}(e) \leq \bar{h}(u)\|e\|^{2}-c \bar{h}(u)\|C e\|^{2}
$$

Instead of proceeding like Tsinias, we remark that (18a) can also be written as [20]:

$$
\dot{V}(e) \leq \bar{h}(u)\left(1-c\left\|C^{T} C\right\|\right)\|e\|^{2}
$$

It is easy to see that with $c>\frac{1}{\left\|C^{T} C\right\|}$, the initial condition is verified.

To summarize, the existence of $P$, a symmetrical positive definite matrix verifying equation (10), and of $h(u)$ verifying (18) are the two conditions needed to design the state observer (2). The block diagram of the resulting nonlinear observer-based residual generation is shown in figure 1 where the time invariant matrix $R(u)$ has to be determined using the preceding algorithm. This will be illustrated by an example in the last section of the paper. 


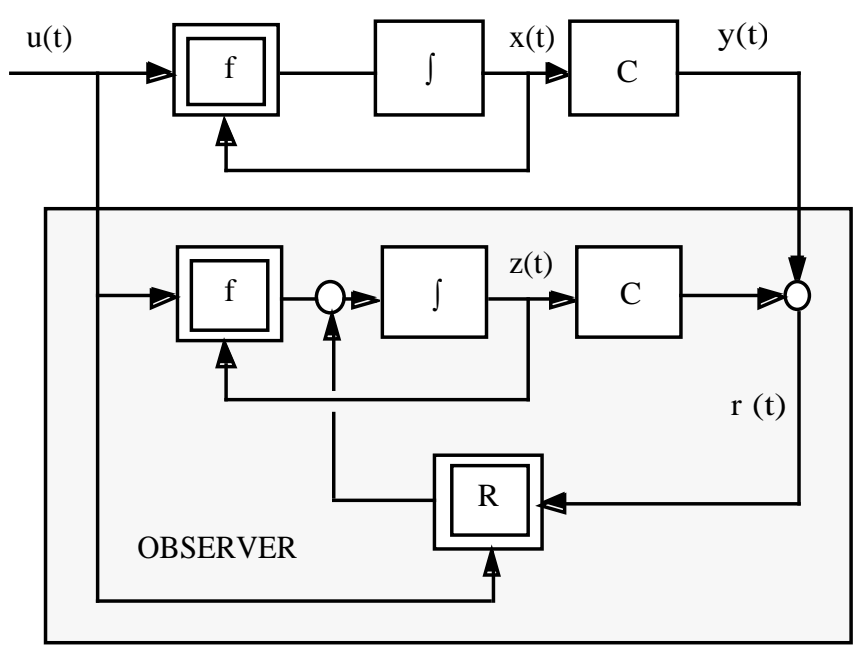

Fig. 1: observer-based residual generation

\section{DEDICATED NON LINEAR OBSERVER SCHEME (DNOS)}

The basic idea of observer approach is to reconstruct the state and output of the process under consideration and then analyze the output estimation error. It is worthwhile recalling here the differential equation governing the dynamics of the output estimation error:

$$
\dot{e}=\left(\frac{\partial f(z, u)}{\partial z}-R(u) C\right) e
$$

In the presence of process or sensor faults, equation (1) may be modified as follows:

$$
\begin{array}{ll}
x=f(x, u)+f_{1} & x \in R^{n}, u \in R^{m} \\
y=C x+f_{2} & y \in R^{p}
\end{array}
$$

where $f_{1}(t)$ and $f_{2}(t)$ represent process and sensor faults respectively. In this case, the state estimation error dynamic is given by:

$$
\dot{e}=\left(\frac{\partial f(z, u)}{\partial z}-R(u) C\right) e+f_{1}-R(u) f_{2}
$$

Since the output estimation error $r(t)=C e(t)$ is a function of $f_{1}(t)$ and $f_{2}(t)$, it can be used as a residual for indicating that a fault has occurred. It is clear from equation (22) that the output estimation error is affected by the faults. The system described by this equation is asymptotically stable, since the stability conditions of the observers are fulfilled. In the ensuing development, we shall limit our attention to sensor and actuator faults only. Generally, fault detection is achieved by comparing the residuals (normalized by their variance) to a specified threshold. To be more precise, the observer has to be designed to facilitate faults isolation. A well-known approach for sensor fault isolation based on dedicated observers scheme [21] may be extended here to the nonlinear case. Each observer is driven by the input vector $u$ and the output of a single dedicated sensor. The complete output (or part of it, for systems that are not completely observable) is estimated and the corresponding residuals are generated and analyzed. If, for example, a sensor fails, the corresponding estimated output will be erroneous. Such a sensor failure can easily be detected and localized using a suitable logic function.

In order to increase the robustness of such an observerbased FDI scheme, one can increase the number of inputs of the different observers; this constitutes the so-called generalized observer scheme introduced by Frank [22] for linear systems. This can directly be extended to nonlinear systems. The solution consists of using the $i$ th observer driven by all but the ith sensor so that a fault on sensor $i$ affects all but the $i$ th residual. With $p$ observers, we then obtain $q$ residuals which allow a unique fault isolator.

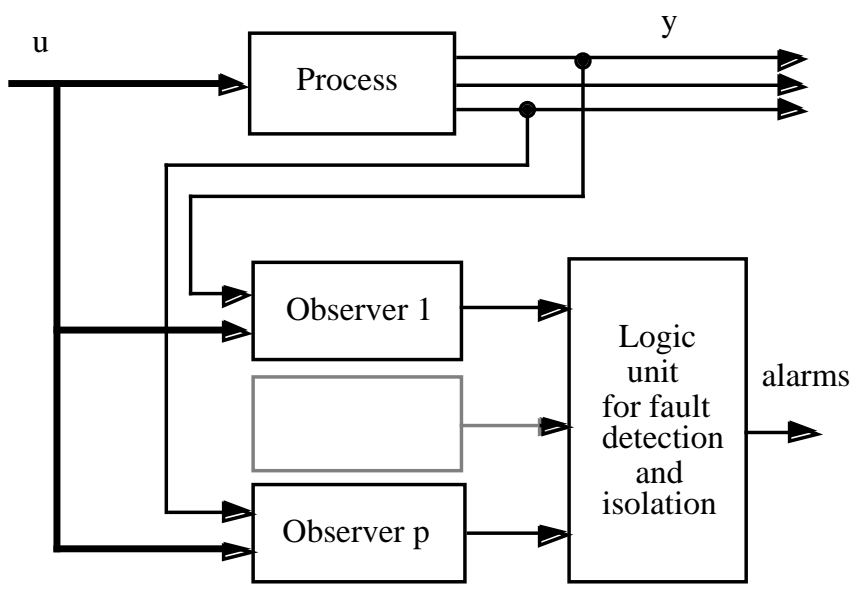

Fig. 2: observer scheme for residual generation and fault isolation

\section{RESIDUAL ANALYSIS}

As previously mentioned, the residual vector results from the combined influence of faults. If there is no noise on the measured variables, any non zero residual is an indication of a fault. However, in a practical situation, the assumption of zero noise is not realistic. Hence we need to separate the influence of faults on residuals from that of noise. This is generally achieved through a statistical test based on the hypothesis that the noise is randomly distributed with zero mean and a given standard deviation.

The most common approach consists in testing the residuals with a separate test applied to each element of the residual vector. A Boolean signature vector is then formed whose entries are set to 1 if the test has fired and 0 otherwise. It is then possible to compare the experimental signature with all the predetermined theoretical signatures corresponding to the different fault situations. Another approach to test the residuals consists of defining a scalar statistic which is simply the sum of squares of the residuals (with the 
possibility of weighting the different residuals). As it is well known, in the linear case, when the noise is normally distributed and when there are no faults, this scalar follows a chi-square distribution; then the experimental value of this statistic can then be compared, for a given level of confidence, to a theoretical value. In the nonlinear case, this distribution does not hold, however, it is possible to use the sum of squares of the residuals as a test variable, the magnitude of which being related to those of faults. This test has the advantage of being easy to apply and to detect the existence of a fault; the isolation of the faults is not directly possible however. This is a major problem for isolation technique to solve. The associated algorithm must be designed to facilitate the recognition of specific failure types. The answer lies in the residual generation; through the use of dedicated observers it is possible to have structured residuals which are sensitive to specific faults. Generally speaking, isolation can be improved by desensitizing residuals with respect to certain faults. In the case of sensor faults isolation, this may be achieved by designing the observers in such a way that each of them uses a specific sensor output.

\section{EXAMPLE}

Let us consider a system governed by the following differential equations:

$$
\begin{aligned}
& x=f(x, u) \\
& y=C x+f
\end{aligned}
$$

where $f$ is a vector of sensor faults, with:

$$
\begin{gathered}
f(x, u)=\left(\begin{array}{c}
x_{2} \\
-\sin \left(x_{1}\right)+u \cos \left(x_{1}\right)-0.4 x_{2} x_{3} \\
\frac{1}{1+x_{2}^{2}}-x_{3}
\end{array}\right) \\
C=\left(\begin{array}{lll}
1 & 0 & 1 \\
0 & 1 & 1
\end{array}\right) \text { and } u \in[-1,1]
\end{gathered}
$$

\section{A. A global nonlinear observer}

Following the proposed method, the first step is to find a positive definite matrix such that for $e \in \operatorname{Ker}(C)$, we have the inequality:

$$
\dot{V}(e)=e^{T} P \frac{\partial f(z, u)}{\partial z} e<0
$$

From $C e=0$, we derive the relation: $e_{2}=e_{1}$ and $e_{3}=-e_{1}$. It is easily shown that the following positive definite matrix $P$ verifies the above inequality.

$$
P=\left(\begin{array}{ccc}
.5 & 0 & 0 \\
0 & .1 & 0 \\
0 & 0 & 2.7
\end{array}\right)
$$

The next step is to find a positive function $\bar{h}(u)$ and a constant $c$ verifying equation (15). One choice is to take $\bar{h}(u)$ $=5$ and $c=0.35$.

$$
\left|e^{T} P \frac{\partial f(z, u)}{\partial z} e\right|-h(u)\|e\|^{2} \text { be fulfilled. }
$$

The observer is then described by the equations:

$$
\begin{aligned}
& \dot{z}=f(z, u)+1.75 C^{T}\left(y-y_{e}\right) \\
& y_{e}=C z
\end{aligned}
$$

Here, our aim is to detect sensor faults. Figure 3 shows the input signal $u(t)$. In this example, a fault is simulated between $4 \mathrm{sec}$. and $6 \mathrm{sec}$. on the first sensor and between $13 \mathrm{sec}$. and $15 \mathrm{sec}$. on the second. We then calculate the output residuals $r(t)$ defined as $r(t)=y(t)-y_{e}(t)$. Figure $4 \mathrm{a}$ and $4 \mathrm{~b}$ show respectively the first and the second output, and their corresponding estimates and residuals: after a transient due to arbitrary initial conditions applied to the observer, the residuals are centred at the origin in the absence of fault. The faults are simultaneously accentuated in both residuals making it impossible to know which of the sensors is faulty.

\section{B. Example of DNOS observer}

Nonlinear observer dedicated to $y_{1}$

The observer is controlled by the input $u$ and the first sensor output $y_{1}$. As the system is observable, the two outputs may be reconstructed and the two residuals generated. In this case, a fine reconstruction of the state would result in the fact that only $r_{l}(t)$ will be sensitive to a fault on $y_{2}$.

For the design purpose, let us consider the observation matrix $C_{1}=\left(\begin{array}{lll}1 & 0 & 1\end{array}\right)$ and $e$ such that $e \in \operatorname{Ker}\left(C_{1}\right)$. From $C_{1} e=0$, we derive the relation $e_{3}=-e_{1}$. The inequality:

$$
e^{T} P \frac{\partial f(z, u)}{\partial z} e<0
$$

holds with:

$$
P=\left(\begin{array}{lll}
1 & 0 & 0 \\
0 & .5 & 0 \\
0 & 0 & 2
\end{array}\right)
$$


We then select $h(u)=8.75$ to respect the constraint $\left|e^{T} P \frac{\partial f(z, u)}{\partial z} e\right|<h(u)\|e\|^{2}$, where $c$ is chosen greater than 0.5. A DNOS observer is designed with $c=1$.

$$
\begin{aligned}
& \dot{z}=f(z, u)+0.5 C^{T}\left(y_{1}-C_{1} z\right) \\
& y_{e}=C z
\end{aligned}
$$

Comparing figure $5 \mathrm{a}$ and $5 \mathrm{~b}$, on time interval from 13 to 15 seconds reveals that the observer input is fault free. That means that the system's state is correctly estimated. It also means that $y_{1} e$ and $y_{2} e$ are correctly reconstructed; residual $r_{1}$ only remains fault sensitive with regards to the second sensor. The comparison of the evolution of $r_{1}$ and $r_{2}$, on time interval from 4 to 6 seconds, shows that they are both affected by the first sensor fault in this observer scheme.

\section{Nonlinear observer dedicated to $y_{2}$}

The second output helps to reconstruct the system state with dedicated nonlinear observer scheme. Results are interpreted in an analogous fashion as in the preceding case: the first residual is sensitive to faults due to both sensors while the second is sensitive to fault due to the second sensor only. Figure $6 \mathrm{a}$ and $6 \mathrm{~b}$ show respectively the residual $r_{l}$, the output $y_{1}$, the output estimate $y_{1 e}$ and the residual $r_{2}$, the output $y_{2}$, the output estimate $y_{2} e$ for fault simulated on the first and second sensor.

We conclude that the observer controlled by the input $u$ and all the sensor outputs is able to detect a sensor fault but not to localize it. Localization of faults necessitates use of dedicated observers which yield fault decoupled residuals with particular geometric fault direction.
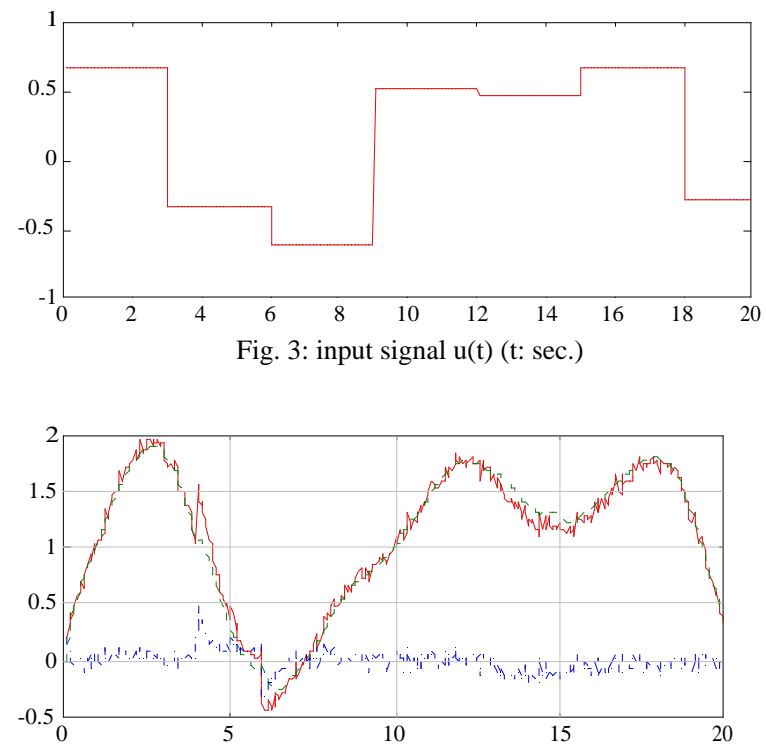

Fig. 4a: (Nonlinear observer scheme results) output $\mathrm{y}_{1}$, estimate $\mathrm{y}_{\mathrm{e} 1}$ and residual $\mathrm{r}_{1}$.

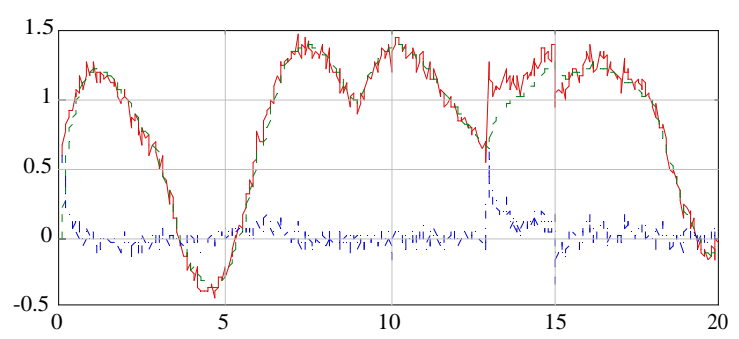

Fig. 4b: (Nonlinear observer scheme results output $\mathrm{y}_{2}$, estimate $\mathrm{y}_{\mathrm{e} 2}$ and residual $\mathrm{r}_{2}$.

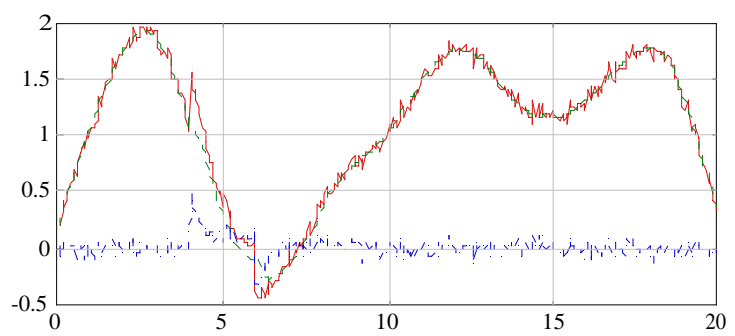

Fig. 5a: (Dedicated nonlinear observer scheme 1) output $\mathrm{y}_{1}$, estimate $\mathrm{y}_{\mathrm{e} 1}$ and residual $\mathrm{r}_{1}$.

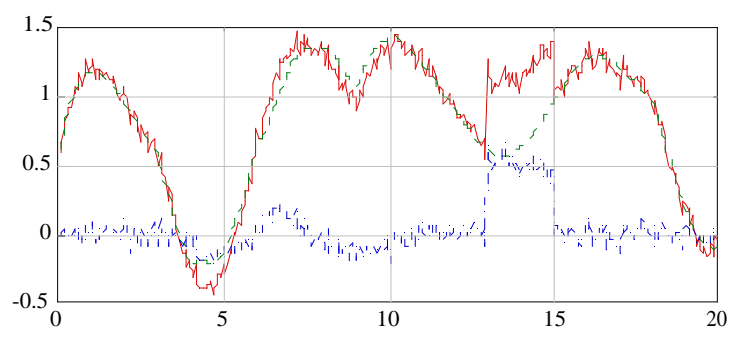

Fig. 5b: (Dedicated nonlinear observer scheme 1) output $\mathrm{y}_{2}$, estimate $\mathrm{y}_{\mathrm{e} 2}$ and residual $\mathrm{r}_{2}$.

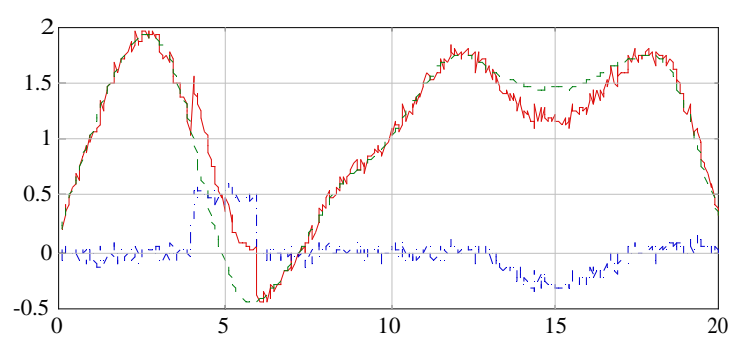

Fig. 6a: (Dedicated nonlinear observer scheme 2) output $\mathrm{y}_{1}$, estimate $\mathrm{y}_{\mathrm{e} 1}$ and residual $\mathrm{r}_{1}$.

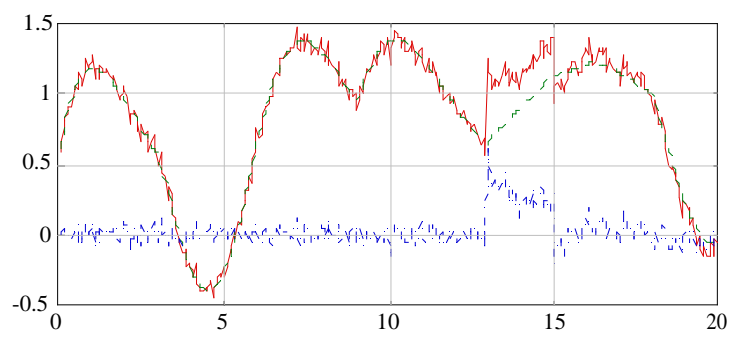

Fig. 6b: (Dedicated nonlinear observer scheme 2) output $\mathrm{y}_{2}$, estimate $\mathrm{y}_{\mathrm{e} 2}$ and residual $\mathrm{r}_{2}$. 


\section{CONCLUSION}

In this paper, we have discussed the analytical redundancy approach to FDI in nonlinear dynamic systems. It has been pointed out how to design an observer with good properties for fault detection. Simulation and experimental results have shown how to apply the dedicated nonlinear observer scheme to the isolation of sensor faults. This observer design solve one of the problems of robustness with respect to nonlinearities in fault detection systems. In practice, it concerns a very large class of nonlinear systems and particularly bilinear systems.

\section{REFERENCES}

[1] D. Luenberger. Observers for multivariable systems. IEEE Trans. on Auto. Control, 11, p. 190-197, 1966.

[2] O'Reilly. Observers for linear systems. Academic Press, 1983.

[3] K. S. Narendra and L. Valavani. Stable adaptative observers and controlers. IEEE Proc. 64, p. 1198-1208, 1976.

[4] G. Kreisselmeier. The generation of adaptive law structures for globally convergent adaptive systems. IEEE Trans. on Auto. Control, 24, p. 510-512, 1979.

[5] G. Kreisselmeier. Adaptive observers with exponential rate of convergence. IEEE Trans. on Auto. Control, 22, p. 2-8, 1977.

[6] J.F. Magni and P. Mouyon. A generalized approach to observers for fault diagnosis. Proc. of the 30th IEEE Conf. on Decision and Control, Brighton, 1991.

[7] N. Viswanadham and R. Srichander. Fault detection using unknown input observer. Control Theory and Advanced Technology, 3, p. 91-101, 1987.

[8] B.L. Walcott and S.H. Zak. Observation of dynamical systems in the presence of bounded nonlinearities/ uncertainties. Proc. of 25th IEEE Conf. on Decision and Control, Athens, 1986.

[9] B.L. Walcott and S.H. Zak. State observation of nonlinear uncertain dynamical systems. IEEE Trans. on Auto. Control, 32, p. 166-170, 1987.

[10] G. Bastin, M.R. Gevers. Stable adaptive observers for nonlinear time-varying systems. IEEE Trans. on Auto. Control, 33 (7), p. 650-658, 1988.
[11] R. Marino. Adaptive observers for single output nonlinear systems. IEEE Trans. on Auto. Control, 35 (9), p. 1054-1058, 1990.

[12] X.H. Xia and W.B. Gao. On exponential observer for nonlinear systems. Systems and Control Letters, 11, p. 319-325, 1988.

[13] A.J. Krener and A. Isodori. Linearization by output injection and nonlinear observers. Systems and Control Letters, 3, p. 47-52, 1983.

[14] R.J. Patton, P.M. Frank and R.N. Clark. Fault diagnosis in dynamic systems. Prentice Hall, 1989.

[15] D. Hengy and P.M. Frank. Component failure detection via nonlinear state observers. Proc. of the IFAC workshop on fault detection and safety in chemical plants, Kyoto, 1986.

[16] R. Seliger and P.M. Frank. Robust component fault detection and isolation in nonlinear dynamic systems using nonlinear unknown input observers. IFAC Symp. Safeprocess, Baden-Baden, 1991.

[17] K. Ogata, Modern control engineering. Englewood Cliffs, NJ, Prentice Hall, 1970.

[18] M. Corless, F. Garofalo, G. Leitmann. Garanteeing ultimate boundedness and exponential rate of convergence for a class of uncertain systems. Robustness in identification and control, Plenum Press, 1988.

[19] J. Tsinias. Observer design for nonlinear systems. Systems and Control Letters, 13, p. 135-142, 1989.

[20] K. Adjallah. Contribution au diagnostic des systèmes par observateurs d'état. Diagnostic d'un train de laminage à chaud et supervision d'une installation de séchage. Thèse de doctorat de l'Institut National Polytechnique de Lorraine, France, 1993.

[21] R.N. Clark. Instrument fault detection. IEEE Trans. on Aerosp. and Electr. System, 14 (3), p. 456-465, 1978.

[22] P.M. Frank. Fault diagnosis in dynamic systems via state estimation - a survey. In System Fault diagnostics. Reliability and Related Knowledge-based Approaches, S. Tzafestas e.a. (Eds), Dr. Reidel Publ. Comp., Dordrecht , 1, p. 35- 98, 1987. 